\title{
Modeling Of Phasor Measurement Unit
}

\author{
B. Ramesh \\ Assistant Professor \\ EEE department, V. R. Siddhartha Engineering College \\ S. N. V. S. K. Chaitanya \\ Assistant Professor \\ EEE department V. R. Siddhartha Engineering College
}

\begin{abstract}
For real time monitoring of power system it has become inevitable to determine the phase angle along with magnitude of voltage and current waveforms. Phasor Measurement Unit is the only device which measures phase angle directly. It measures the voltage and current phasors at different buses of a power system synchronized by a common timing signal. The measured data is received by the central server through fibre optic communication. This paper describes the Phasor Measurement Unit modeling using MATLAB SIMULINK.
\end{abstract} Index Terms: Phasor Measurement Unit (PMU), Global Positioning System (GPS),
Discrete Fourier Transform (DFT)

\section{INTRODUCTION}

State Estimation is of great importance in real time monitoring of power systems. Supervisory Control and Data Acquisition (SCADA) systems were used to monitor important system data such as voltage, current and line flows, updates measurement with a sampling frequency in the range of a few seconds[1]. However, there is now a need for faster sampling and synchronization of measurements. PMUs are usually installed at substations and are responsible for calculating/reporting voltage and current phasors. Using GPS time as reference, signals measured at different buses can be synchronized accurately with error as low as one micro second[3]. PMUs provide accurate data which enables the power system engineers to analyze different disturbances and load fluctuations so that power system stability, reliability can be improved and possibility of blackouts can be reduced to maximum extent .

\section{PMU ARCHITECTURE}

A PMU provides positive sequence voltage and current phasors at different network buses of power system[2]. The phasors may be single phase or symmetrical component values. The PMU provides a real-time data and it may also provide measurements of other analog and digital signals, including raw synchronized samples, and may also record data locally. The PMU perform functions, such as relaying, metering and fault signal recording. Likewise, the PMU function shall not interfere with the performance of other functions. 
The main components of PMU are:

- Analog Inputs

- GPS receiver

- Phase locked oscillator

- $\mathrm{A} / \mathrm{D}$ converter

- Anti-aliasing filters

- Phasor micro-processor

- Modem

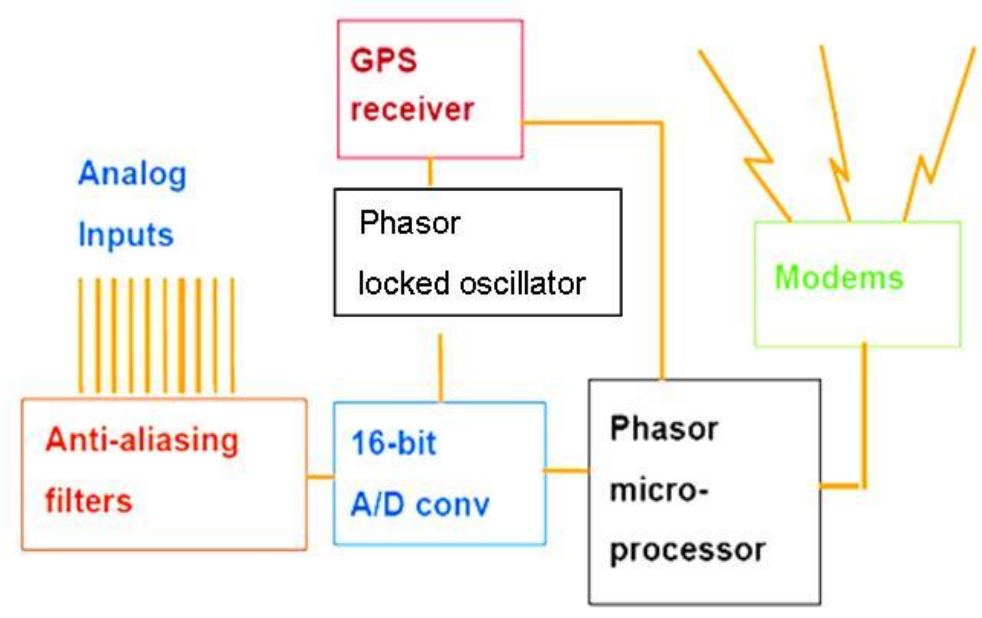

Fig. 1 Block Diagram of PMU

\section{Analog inputs:}

Current and potential transformers are employed at substation for measurement of voltage and current. The analog inputs to the PMU are the voltages and currents obtained from the secondary winding of potential and current transformers.

\section{$\underline{\text { Anti-aliasing filters: }}$}

Anti-aliasing filter is an analog low pass filter which is used to filter out those components from the actual signal whose frequencies are greater than or equal to half of nyquist rate to get the sampled waveform[3]. Nyquist rate is equal to twice the highest frequency component of input analog signal. If anti aliasing filters are not used, error will be introduced in the estimated phasor.

\section{Phase lock oscillator:}

Phase lock oscillator along with Global Positioning System reference source provides the needed high speed synchronized sampling; Global Positioning System (GPS) is a satellite-based system for providing position and time. 


\section{$\underline{\text { A/D converter: }}$}

It converts the analog signal to the digital signal. Quantization of the input involves in ADC that introduces a small amount of error. The output of ADC is a sequence of digital values that convert a continuous time and amplitude analog signal to a discrete time and discrete amplitude signal. It is therefore required to define the rate at which new digital values are sampled from the analog signal. The rate of new values at which digital values are sampled is called the sampling rate of the converter.

\section{Global Positioning System:}

The synchronized time is given by GPS uses the high accuracy clock from satellite technology. The first GPS system was developed by United States, Department of Defence. Without GPS providing the synchronized time, it is hard to monitor whole grid at the same time. The GPS system consists of 24 satellites in six orbits at an approximate altitude of 10,000 miles above the surface of the earth. They are thus approximately at one half the altitudes corresponding to a geosynchronous orbit. The positioning of satellite in orbits and the positioning of orbital plane is such that at any given instant at least six satellites visible at most location on earth. Often as many as 10 satellites may be available for viewing[4]. The civilian-use channel of the GPS transmits positional coordinates of the satellite from which the location of a receiver station on the earth could be determined. In addition, the satellites transmit a one pulse per second signal. This pulse as received by any receiver on earth is coincident with all other received pulses within 1 microsecond and often in practice is found to be much more accurate[5]. The GPS satellites provide a very accurate time synchronization signal, available, via an antenna input, throughout the power system. This means that that voltage and current recordings from different substations can be directly displayed on the same time axis and in the same phasor diagram.

\section{Processor:}

The microprocessor calculates positive-sequence estimates of all the current and voltage signals using the DFT techniques. Certain other estimates of interest are frequency and rate of change of frequency measured locally, and these also are included in the output of the PMU. The timestamp is created from two of the signals derived from the GPS receiver. The time-stamp identifies the identity of the "universal time coordinated (UTC) second and the instant defining the boundary of one of the power frequency periods.

\section{Modem:}

A device that modulates an analog carrier signal and encodes digital information from the signal and can also demodulate the signal to decode the transmitted information from signal is called modem [1]. The objective of modem is to produce a signal that can be transmitted and decoded to make a replica of the original digital data. Modem can be used with no means of transmitting analog signals

\section{MATLAB MODELING OF PMU}

Input voltage signal 
The input signal is an analog voltage signal. Here a sine wave with the parameters of amplitude $11000 \mathrm{~V}, \mathrm{f}=50 \mathrm{~Hz}$, phase of $\pi / 6$ radians with a sample time of $0.1 \mathrm{msec}$. The wave considered is time based wave form.

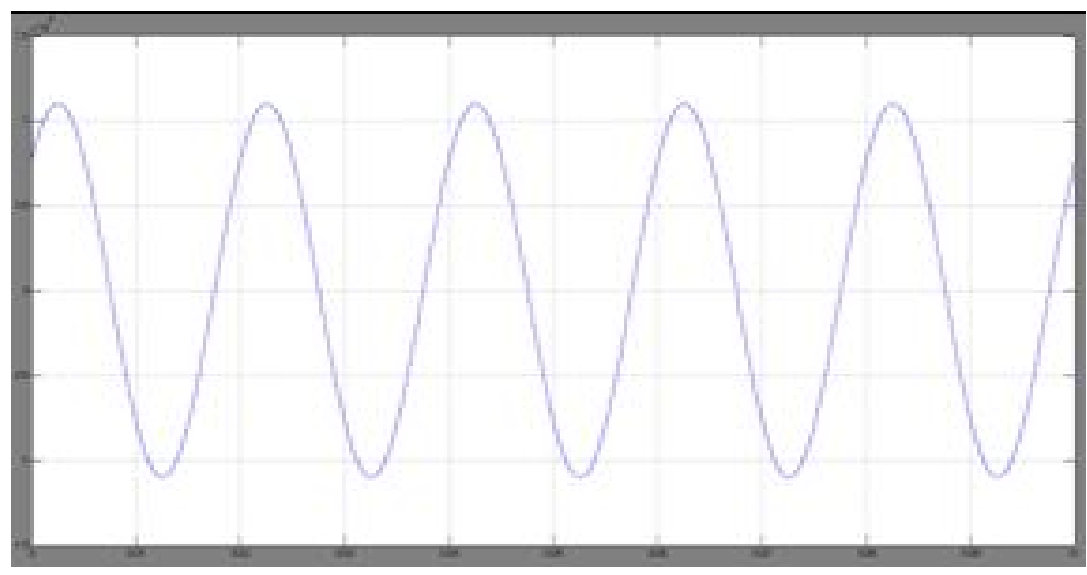

Fig. 2 Input signal

\section{$\underline{\text { Analog to digital converter }}$}

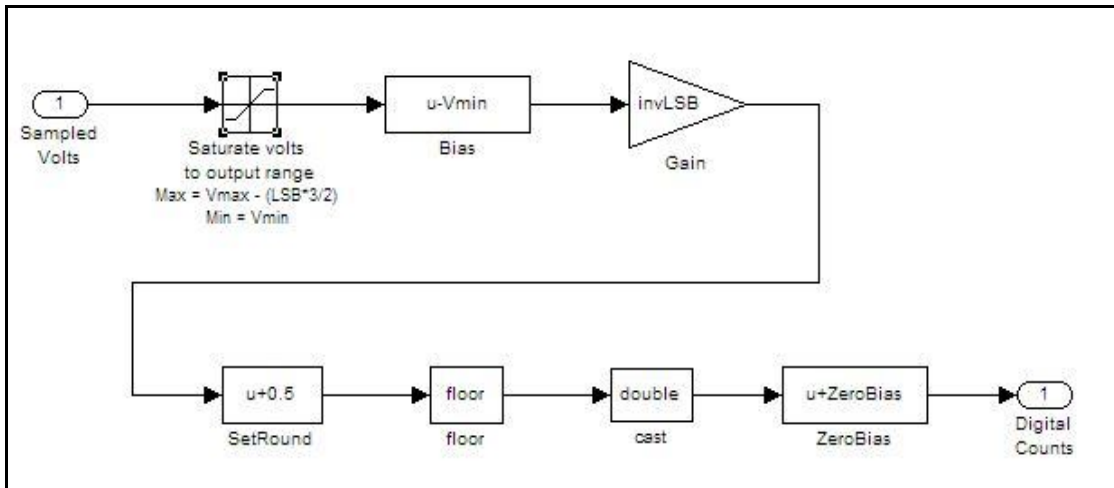

Fig. 3 Simulink model of analog to digital converter 


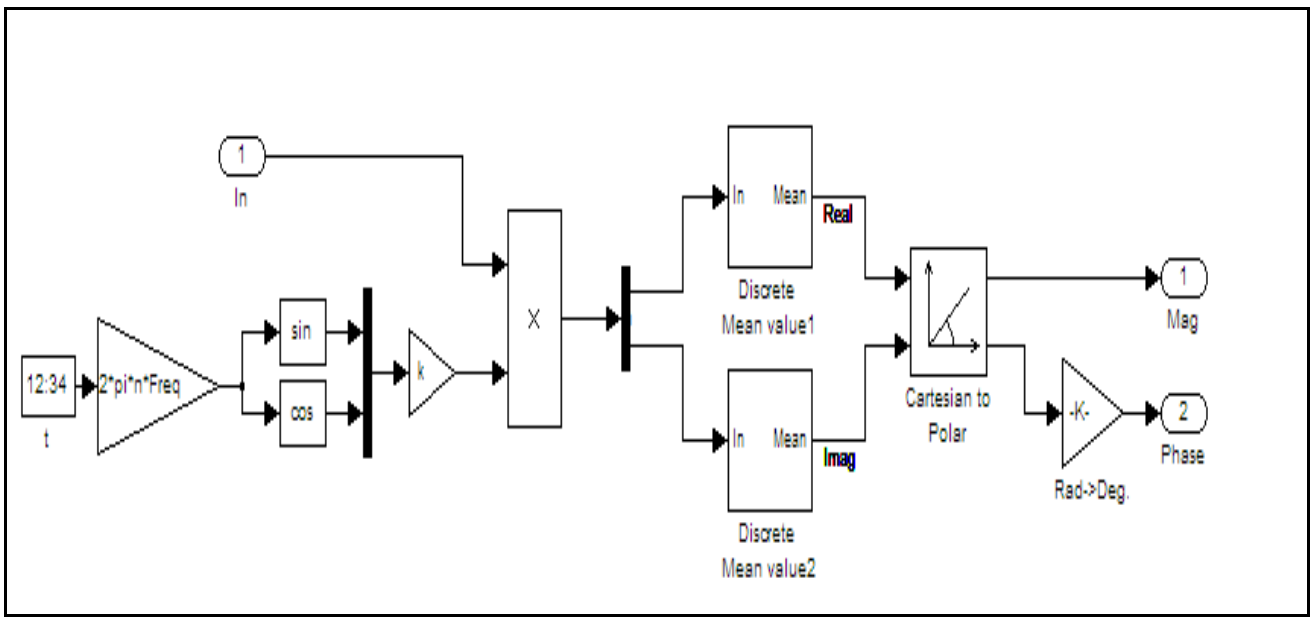

Fig. 4 Simulink model of Discrete Fourier Transform

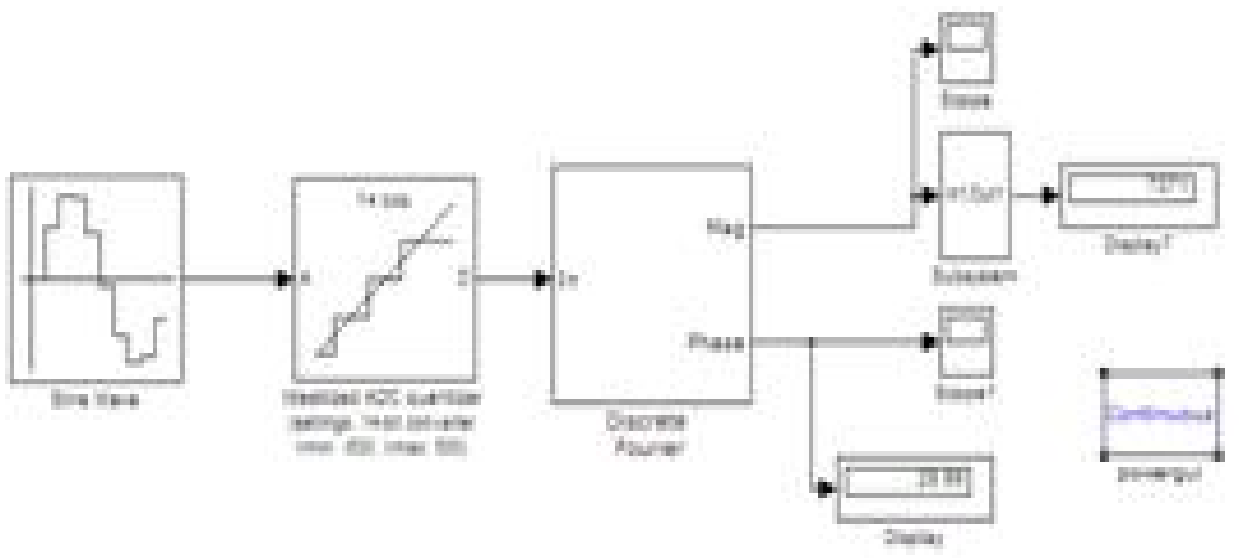

Fig. 5 Simulink model of Phasor Measurement Unit

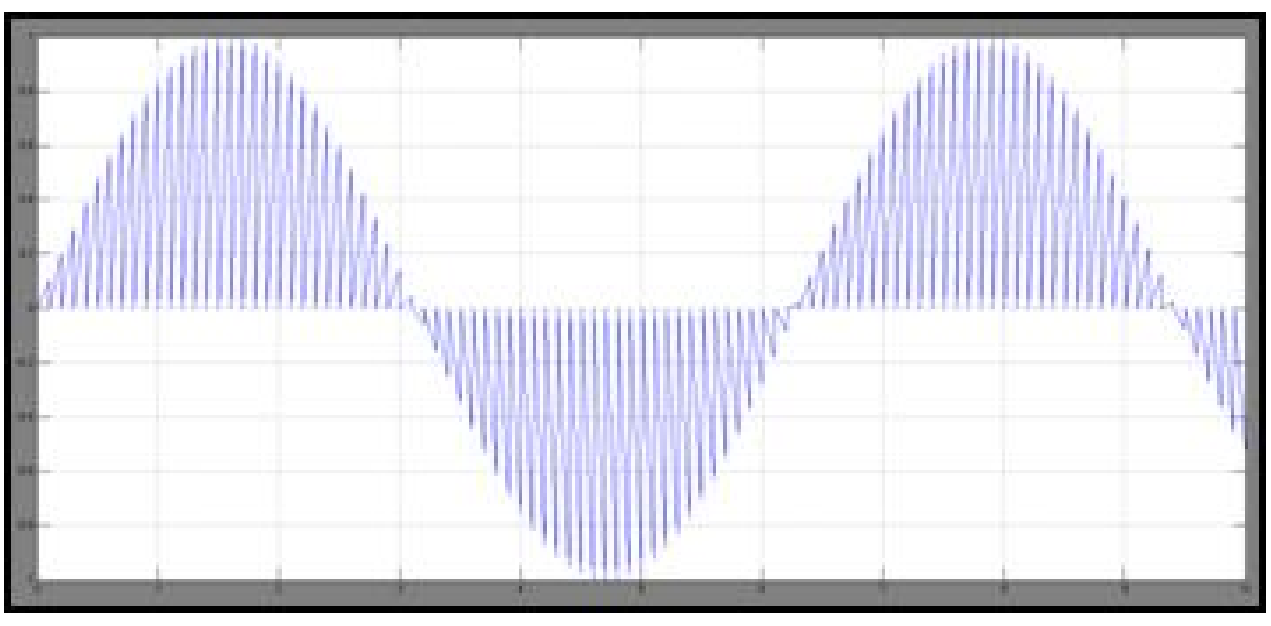

Fig. 6 Sampled Signal 


\section{RESULT}

\begin{tabular}{|c|c|c|c|c|}
\hline & $\begin{array}{c}\text { Input RMS } \\
\text { voltage }\end{array}$ & $\begin{array}{c}\text { Phase } \\
\text { (in deg) }\end{array}$ & $\begin{array}{c}\text { Output RMS } \\
\text { Voltage }\end{array}$ & $\begin{array}{c}\text { Phase } \\
\text { (in deg) }\end{array}$ \\
\hline 1 & $11 \mathrm{kV}$ & 75 & $10.988 \mathrm{kV}$ & 74.95 \\
\hline 2 & $6.6 \mathrm{kV}$ & 30 & $6.589 \mathrm{kV}$ & 29.54 \\
\hline 3 & $3.3 \mathrm{kV}$ & 45 & $3.287 \mathrm{kV}$ & 44.63 \\
\hline
\end{tabular}

\section{CONCLUSION}

Modeling of PMU using MATLAB Simulink has been presented. The modeling of PMU is done with sampled sine wave, a 14-bit ADC consisting of quantizer and sample/hold circuit, DFT for phasor estimation.

\section{REFERENCES}

[1] A J. De La Ree, V. Centeno, J. S. Thorp, A. G. Phadke, "Synchronized Phasor Measurement Applications in Power Systems", IEEE Transactions on Smart Grid, vol. 1, no. 1, pp. 20-27, June 2010.

[2] IEEE Standard for Synchrophasors for power Systems, IEEE Standard C37.118-2005 (Revision of IEEE Std 1344-1995).

[3] Jaime De La Ree, Virgilio Centeno, J.S. Thorp, A. G. Phadke "Synchronized Phasor Measurement Applications in Power Systems", 'IEEE transactions on smart grid, vol. 1, no. 1,Jjune 2010'

[4] A. Phadke, J. Thorp, M. Adamiak ,"A New Measurement Technique for Tracking Voltage Phasor, Local System Frequency, and Rate of Change of Frequency"; IEEE Trans. vol. PAS-102 no. 5, May 1983, pp 1025-1038

[5] B.PhaniRanga Raja, B.Ramesh " Synchronized Phasor Measurements Based Power System Dynamic State Estimation”, IEEJ Vol. 6 (2015) No.8, pp. 2018-2023 ISSN 2078-2365. 